\title{
Depression and low physical activity are related to sarcopenia in hemodialysis: A single-center study
}

\author{
Kornanong Yuenyongchaiwat ${ }^{\text {Corresp., } 1,2}$, Sasikan Jongritthiporn ${ }^{1}$, Kasarn Somsamarn $^{1}$, Oranat Sukkho ${ }^{1}$, Sasipim \\ Pairojkittrakul $^{3}$, Opas Traitanon ${ }^{4}$ \\ ${ }^{1}$ Department of Physiotherapy/ Faculty of Allied Health Sciences, Thammasat University, Pathumthani, Thailand \\ 2 Thammasat University, Research Unit in Physical therapy in respiratory and cardiovascular systems, Pathumthani, Pathumthani, Thailand \\ 3 Nephrology clinic, Thammasat University hospital, Pathumthani, Thailand \\ 4 Division of Nephrology, Department of Internal medicine, Faculty of Medicine, Thammasat University, Pathumthani, Thailand \\ Corresponding Author: Kornanong Yuenyongchaiwat \\ Email address: ykornano@tu.ac.th
}

Background: The number of patients who suffer from chronic renal failure (CRF) has widely increased worldwide. Patients with advanced stages of CRF experience a gradual and progressive loss of muscle and fat mass leading to decreased physical activity and mental health problems. The loss of muscle mass in CRF might contribute to the development of sarcopenia. Therefore, this study aimed to explore the prevalence of sarcopenia and to determine the relationship of physical activity and mental state of depression with sarcopenia in hemodialysis patients. Methods: A cross-sectional study was designed with a total of 104 male and female with a minimum age of 35 years. Based on the guidelines of the Asian Working Group for Sarcopenia in 2019, gait speed, muscle mass, and handgrip were used to define sarcopenia. In addition, participants were requested to perform a set of questionnaires to evaluate their physical activity and state of depression. Logistic regression analyses were used to explore the risk factors of sarcopenia. Results: Thirty-four (32.69\%) of 104 participants had sarcopenia. Compared to the 70 individuals without sarcopenia, they had a low physical activity and a high depression score (ps<.05). Furthermore, low physical activity and high depression scores in combination with sarcopenia were associated with an increased mortality risk. Low physical activity and high depression scores were also independently associated with sarcopenia in hemodialysis patients after controlling for age (Odds ratio $=3.23$, and 4.92 , respectively). 
1 Depression and low physical activity are related to

\section{Authors' names and institutional affiliations;}

Kornanong Yuenyongchaiwat ${ }^{*}$, PT, Ph.D., Sasikan Jongritthiporn1 PT, BSc., Kasarn

Somsamarn1 PT, BSc., Oranat Sukkho1 PT, MSc., Sasipim Pairojkittrakul2, M.Sc., Opas

Traitanon3, MD.

${ }^{1}$ Physiotherapy Department, Faculty of Allied Health Sciences, Thammasat University

${ }^{2}$ Nephrology clinic, Thammasat University hospital

${ }^{3}$ Division of Nephrology, Department of Internal medicine, Faculty of Medicine, Thammasat University

\section{Corresponding author:}

Assoc. Prof. Kornanong Yuenyongchaiwat, Ph.D.

${ }^{1}$ Physiotherapy Department, Faculty of Allied Health Sciences, Thammasat University, 99 Moo 18, Paholyothin Road, Klong Luang, Rangsit, Pathumthani, 12120, Thailand.

Tel +66 (0)29869213, E-mail: ykornano@tu.ac.th

2 Thammasat University Research Unit in Physical therapy in respiratory and cardiovascular systems, Pathumthani, Thailand. 


\section{Abstract}

24

25

26

27

28

29

30

31

32

33

34

35

36

37

38

39

40

41

42

43

44

45

46

47

Background: The number of patients who suffer from chronic renal failure (CRF) has widely increased worldwide. Patients with advanced stages of CRF experience a gradual and progressive loss of muscle and fat mass leading to decreased physical activity and mental health problems. The loss of muscle mass in CRF might contribute to the development of sarcopenia. Therefore, this study aimed to explore the prevalence of sarcopenia and to determine the relationship of physical activity and mental state of depression with sarcopenia in hemodialysis patients.

Methods: A cross-sectional study was designed with a total of 104 male and female with a minimum age of 35 years. Based on the guidelines of the Asian Working Group for Sarcopenia in 2019, gait speed, muscle mass, and handgrip were used to define sarcopenia. In addition, participants were requested to perform a set of questionnaires to evaluate their physical activity and state of depression. Logistic regression analyses were used to explore the risk factors of sarcopenia.

Results: Thirty-four (32.69\%) of 104 participants had sarcopenia. Compared to the 70 individuals without sarcopenia, they had a low physical activity and a high depression score $(\mathrm{ps}<.05)$. Furthermore, low physical activity and high depression scores in combination with sarcopenia were associated with an increased mortality risk. Low physical activity and high depression scores were also independently associated with sarcopenia in hemodialysis patients after controlling for age (Odds ratio $=3.23$, and 4.92, respectively).

Keywords: Physical activity; Depression; Sarcopenia; Hemodialysis; Prevalence 
48

49

50

51

52

53

54

55

56

57

58

59

60

61

62

63

64

65

66

67

68

69

70

71

72

73

74

75

76

77

78

79

80

81

82

83

84

85

86

87

88

89

90

91

92

93

\section{Introduction}

Chronic renal failure (CRF) is a common worldwide health problem including in Thailand. A $17.5 \%$ prevalence of CRF was reported in Thailand with a prevalence of $7.8 \%$ in males and $9.3 \%$ in females at stages 3-5 (Ingsathit et al., 2010). Prevalence of CRF stages 4-5 was increased to $3.6 \%, 7.2 \%$ and $12.7 \%$ at the age of 60,70 , and over 80 years, respectively (Krittayaphong et al., 2017). Also, over 100,000 of the CRF patients in Thailand underwent hemodialysis

(Kanjanabuch et al., 2020). The comorbidity of hemodialysis patients is associated with physical health dysfunction, poor physical activity, and mental health problems. These will lead to a poor quality of life. Regarding depression, it has been reported that mental health complications, in particular higher depressive symptoms, are commonly observed in CRF patients. Furthermore, a systematic review and metanalysis revealed that the relationship between lower albumin in CRF and end stage renal failure is associated with severe depressive symptoms (ascertained by selfreported measures) (Gregg et al., 2020). Therefore, protein-energy wasting was proposed as a potential mechanism underlying the high comorbidity of depression. In addition, hemodialysis patients had a low level of physical activity and more sedentary lifestyle compared with age- and gender-matched individuals without CRF (Gomes et al., 2015). Uremic myopathy that leads to muscle wasting and poor muscle performance has been reported in patients with CRF undergoing hemodialysis (Fahal, 2014). Recently, sarcopenia has been defined in terms of loss of muscle mass and it is categorized into primary and secondary sarcopenia (Cruz-Jentoft et al., 2019). Muscle atrophy, less activity and also reduced physical function has been observed in dialysis patients and might be caused by uremic myopathy (Fahal, 2014). Therefore, the purpose of this study was to explore the prevalence of sarcopenia and the relationships with physical activity and depressive symptoms in Thai CRF patients undergoing hemodialysis.

\section{Materials \& Methods}

This study was carried out according to the principles of the Declaration of Helsinki 1975. The Research Ethics Committee on Human Subject of Thammasat University (approval reference COA No. 117/2562) and the Ethics in Human Research Committee of the Thammasat University Hospital approved the study. Information sheets and the consent form were given to the participating patients prior to the study. The Clinical Trials Registry (TCTR) is TCTR20190911004. The following formula was used to calculate the sample size, $n=N$ / $[1+\mathrm{Ne} 2]$; where $\mathrm{N}=$ total number of hemodialysis patients at the Hemodialysis Center of Thammasat University Hospital, $\mathrm{n}=$ number of samples, and $\mathrm{e}=$ error tolerance $=0.05$. According to a report from the Hemodialysis Center of Thammasat University Hospital in 2018, the number of hemodialysis patients was 140. Therefore, a total of 104 Thai patients who underwent hemodialysis were recruited. The minimum age of the male and female participants was 35 years. These patients required hemodialysis at least two times per week. According to American College of Cardiology and American Heart Association recommendations, resting blood pressure $>180 / 120 \mathrm{mmHg}$ is defined as hypertensive and should be see a doctor (Whelton et al., 2017). In addition, postural orthostatic tachycardia syndrome is assessed by resting heart rate $\geq 120$ beats per min (Schmidt et al., 2017). Therefore, these conditions were excluded in the present study. In addition, participants who had a high fever within 24 hours prior to the test, psychiatric problems or pregnant women were also excluded. Participants were asked to complete a set of questionnaires, i.e., Beck Depression Inventory-II (BDI-II) and Global Physical Activity Questionnaire (GPAQ). BDI-II comprises 21 items with higher scores indicating greater depression. A total score of 13 or less is interpreted as minimal depression. A score between 14 
and 19 is interpreted as mild depression, a score of $20-28$ is interpreted as moderate depression, and a score of 29-63 is interpreted as severe depression (Beck et al., 1996). In addition, the GPAQ was developed by the World Health Organization and it has been commonly used to define physical activity level (Armstrong \& Bull, 2006). A high physical activity is categorized as $\geq 1,500$ MET minutes per week and low physical activity as is defined as $<600$ MET minutes per week (Armstrong \& Bull, 2006). A screening sarcopenia in the present study followed a new version of Asian Working Group for Sarcopenia (AWGS) in 2019 which are poor muscle strength or slow gait speed plus loss of muscle mass (Chen et al., 2019). The applied AWGS 2019 cutoffs for sarcopenia diagnosis were as follows: slow gait speed $(<1.0 \mathrm{~m} / \mathrm{s})$ or poor physical performance (handgrip strength $<28 \mathrm{~kg}$ for men and $<18 \mathrm{~kg}$ for women) plus low muscle mass $\left(<7.0 \mathrm{~kg} / \mathrm{m} 2\right.$ in men and $<5.7 \mathrm{~kg} / \mathrm{m}^{2}$ in women). Therefore, all participants were requested to perform a 6-meter walking test (i.e., gait speed) and a handgrip strength test. A walking speed lower than $1.0 \mathrm{~m} / \mathrm{s}$ was categorized as slow gait speed. In addition, poor muscle strength was categorized as handgrip strength less than $28 \mathrm{~kg}$ in men and less than $18 \mathrm{~kg}$ in women. Participants who had low handgrip strength and/or slow gait speed were required to evaluate the skeletal muscle mass (SMM). The participants were required to perform the bioelectrical impedance analysis (BIA: Omron HBF-375 body composition monitor; Omron Healthcare Co., Ltd., Japan) with body weight, BMI, and SMM also recorded. Skeletal muscle index (SMI) was calculated by SMM in $\mathrm{kg}$ adjusted for the squared height $\left(\mathrm{SMM} / \mathrm{hight}^{2}, \mathrm{~kg} / \mathrm{m}^{2}\right)$. A SMI value of $<7.0 \mathrm{~kg} / \mathrm{m}^{2}$ in men and $<5.7 \mathrm{~kg} / \mathrm{m}^{2}$ in women was defined as low muscle mass.

To compare between sarcopenic and non-sarcopenic patients with hemodialysis, T-test and chisquare test were used. Pearson correlation was used to determine the relationships of sarcopenia. Finally, logistic analysis was performed to explore the independent risk for sarcopenia, a p-value of 0.05 was considered statistically significant. IBM SPSS version 24.0 was used for statistical analyses.

\section{Results}

A total of 104 hemodialysis patients, 54 males and 50 females, were recruited and sarcopenia was found in $34(32.69 \%)$ of them. Classification was due to either poor grip strength $(n=4$, $3.85 \%)$, low gait speed $(\mathrm{n}=7,6.73 \%)$, or concomitant declined muscle strength and low gait speed $(n=23,22.12 \%)$.

The average age of the 104 participants was 59.74(13.62) years. The 34 participants with sarcopenia were observed in advanced age, lower BMI, and longer duration of hemodialysis compared to those without sarcopenia (Table 1). Regarding underlying disease, participants with sarcopenia were significantly affected by a history of diabetes mellitus $(\mathrm{DM})\left(\chi^{2}=5.861, \mathrm{P}=\right.$ $0.018)$. Lower physical activity, higher depression scores and poor cognitive performance were also reported in the sarcopenia group (Table 1).

\section{Insert Table 1 here}

The correlation of risk factors with sarcopenia was examined and the results are presented in Table 2. Risk factors, e.g., physical activity and depression were associated with sarcopenia in hemodialysis patients (Ps $>.05)$. 
140

\section{Insert Table 2 here}

To determine the risk factors for developing sarcopenia, a logistic regression analysis was used. Age, sex, BMI, history of DM, duration of hemodialysis, symptoms of depression and levels of physical activity were explored (Table 3). Depression was found to be a major risk factor (odds ratio (OR) 3.229, 95\% confidence interval (CI) 1.139-9.157, $\mathrm{p}=.028$ ) followed by history of $\mathrm{DM}(\mathrm{OR}=.356,95 \% \mathrm{CI}=0.152-0.832, \mathrm{p}=.017)$, duration of hemodialysis (longer than 10 years $)(\mathrm{OR}=0.316,95 \% \mathrm{CI}=0.111-0.896, \mathrm{p}=.030)$ and low physical activity $(\mathrm{OR}=0.284$, $95 \% \mathrm{CI}=0.121-0.668, \mathrm{p}=.004)$.

\section{Discussion}

Insert Table 3 here
The present study aimed to explore the prevalence of sarcopenia in CRF patients with hemodialysis and to also explore the relationships between sarcopenia, depression, and physical activity. Data from a total of 104 patients were analyzed and sarcopenia was detected in 34 patients $(32.69 \%)$. This prevalence is high compared to the reported $16 \%$ overall prevalence of sarcopenia in elderly from Thailand (Yuenyongchaiwat et al., 2020). The results show that secondary sarcopenia has a higher prevalence rate in CRF patients than primary sarcopenia has in the older adults in Thailand.

Advanced age, long duration of hemodialysis, low physical activity and symptoms of depression were also found associated with sarcopenia in hemodialysis patients. Bataille et al. (2017) found that $31.5 \%$ of hemodialysis patients was defined as sarcopenia and these were older, had longer dialysis, and low BMI. Regarding the European Working Group on Sarcopenia in Older People (EWGSOP) criteria, it was noted that the prevalence of sarcopenia in patients undergoing hemodialysis was 37\% (Kim et al., 2014). A 33.3\% prevalence of sarcopenia was reported in persons older than 60 years (Ren et al., 2016). Mori et al. (2019) reported a $40 \%$ prevalence of sarcopenia in patients undergoing hemodialysis. Here, the present study found the prevalence of sarcopenia was $32.69 \%$ by AWGS definition. Therefore, the reported prevalence of sarcopenia in hemodialysis patients varies somewhat, either due to definition (i.e., the European Working Group on Sarcopenia in Older People; EWGSOP, AWGS), measurement of muscle mass (e.g., DEXA, BIA), or method and cutoff. In all the hemodialysis patients had a prevalence rate of sarcopenia in the range of 31.5-40\% (Bataille et al., 2017; Kim et al., 2014; Mori et al., 2019; Ren et al., 2016). Furthermore, longer duration of hemodialysis was closely associated with higher sarcopenia occurrence in the patients (Ren et al., 2016). CRF in hemodialysis patients has been found together with poor physical function due to loss of muscle strength caused by muscle atrophy and hypoplasia (Fahal, 2013). The mechanism of uremic change might contribute to the decrease in the synthesis of muscle protein and result in uremic sarcopenia (Fahal, 2014).

Regarding the comorbidities, the present study found that a history of DM was associated with sarcopenia in hemodialysis patients. Likewise, Mori, et al. (2019) reported that the DM was an independent factor for sarcopenia in Japanese patients undergoing hemodialysis (OR $=3.11$ ). Association of DM and sarcopenia has been reported in several studies (Kim et al., 2010; Park et al., 2006). Insulin deficiency might be an important contributor to the development of sarcopenia 
185

(Rasmussen et al., 2006; Wang et al., 2016). Mori, et al. (2019) reported the prevalence of $40 \%$ and patients with DM shown a higher risk for sarcopenia than those without history of DM. It is also known that insulin deficiency leads to a reduction in protein synthesis in muscle mass (Mori et al., 2019). In addition, uremic sarcopenia has been proposed as skeletal complication, e.g., muscle wasting, in CRF (Fujita et al., 2006). Mitochondrial dysfunction in skeletal muscle in patients with CRF play an important role in promoting the uremic sarcopenia (Nishi et al., 2020). The induced mitochondrial damage can promote inflammation or cause the release of cytochrome; resulting in apoptosis and reduced ATP synthesis leads to cell damage. Therefore, these cellular processes may develop uremic sarcopenia in CRF patients (Takemura et al., 2020).

The present study found that sarcopenic patients had a lower physical activity compared to nonsarcopenic patients. It is known that loss of muscle mass occurs with ageing and causes primary sarcopenia. However, secondary sarcopenia has been described to be not only due to ageing but also to low physical activity, disease and nutritional factors (Sabatino et al., 2020). Regarding physical inactivity, it is possible to identify an individual who had a sedentary lifestyle, bed rest, for example. In addition, disease conditions such as organ failure disease, inflammatory disease have been observed in secondary sarcopenia. Increased protein degradation such as muscle wasting from CRF leads to protein energy wasting and contributes to malnutrition in patients with CRF. Moreover, decreased protein synthesis has been found in persons with low physical activity which results in muscle disuse and may contribute to sarcopenia (Sabatino et al., 2020). Therefore, both, high protein degradation and low protein synthesis may play a role in the development of sarcopenia in patients with CRF.

Besides, it is unknown whether decreased physical activity causes loss of muscle or muscle loss causes decreased physical activity. Additionally, sarcopenic hemodialysis patients had poor physical performance and/or muscle strength which again might increase physical inactivity and also cause depressive symptoms. Vettoretti et al. (2019) reported a higher prevalence of depression was associated with sarcopenia in CRF patients. It was suggested that these relationships might be explained by physical inactivity and malnutrition. Therefore, reduced physical activity causes depression and vice versa depression causes physical inactivity. In the present study it was found that physical activity was inversely associated with depressive symptoms, i.e., individuals with symptoms of depression had lower physical activity. Further studies need to explore the relationships between depression and physical activity in sarcopenic CRF patients.

Further studies are required to explore potential causative factors and mechanisms for development of sarcopenia in CRF such as inflammatory markers. Besides, an assessment of sarcopenia should be considered in individuals at increased risk for sarcopenia in hemodialysis. Secondary sarcopenia is associated with chronic illness states such as CRF and the present study supports that less physical activity can be observed in sarcopenic hemodialysis patients. It is known that low physical activity is a behavioral risk factor, therefore, the implementation of physical activity should be explored to prevent/treat sarcopenia.

The main limitation of the study was its cross-sectional design, use of a single center and relatively small sample size. Thus, a causal relationship of physical activity and depression with sarcopenia might be difficult to establish. Therefore, the prevalence of sarcopenia in 
231 hemodialysis patients from the study may have limited generalizability. Despite the limitation 232 noted, the study extends pervious research that has been completed in sarcopenia among patients 233 receiving hemodialysis and explore the relationships with physical activity and depression.

\section{Conclusions}

Decreased physical activity might lead to symptom of depression and vice versa depression might contribute be physical inactivity and these also play an important role in sarcopenia among hemodialysis patients.

\section{Acknowledgements}

The authors would like to thank all staff from the Hemodialysis Center at Thammasat University Hospital for their kind support. In addition, we would also like to thank the participants and their caregivers for participating in the study. We would like to pass on our thanks and appreciation to Prof. Nantika Thavichachart, M.D., Department of Psychiatry, Faculty of Medicine, Chulalongkorn University, Thailand for her permission to use the Thai version of Beck Depression Inventory. Finally, the authors gratefully acknowledge the financial support provided by Thammasat University. This study was supported by Thammasat University Research Fund, Contract No. TUFT 016/2563. There is no conflict of interest in this study. 
254

255

256

257

258

259

260

261

262

263

264

265

266

267

268

269

270

271

272

273

274

275

276

277

278

279

280

281

282

283

284

285

286

287

288

289

290

291

292

293

294

295

296

297

298

299

\section{References}

1. Armstrong T. \& Bull F. 2006. Development of the World Health Organization Global Physical Activity Questionnaire (GPAQ). Journal of Public Health 14:66-70.

2. Bataille S, Serveaux M, Carreno E, Pedinielli N, Darmon P, Robert A. 2017. The diagnosis of sarcopenia is mainly driven by muscle mass in hemodialysis patients. Clinical Nutrition 36(6): 1654-1660.

3. Beck AT, Steer RA, Brown G. 1996. Manual for the beck Depression Inventory-II. San Antonio: Psychological Corporation.

4. Chen KL, Woo J, Assantachai P, Auyeung TW, Chou MY, Iijima K, Jang HC, Kang L, Kim M, Kim S, Kojima T, Kuzuya M, Lee JSW, Lee SY, Lee WJ, Lee Y, Liang CK, Lim JY, Lim WS, Pen LN, Sugimoto K, Tanaka T, Won CW, Yamada M, Zhang T, Akishita M, Arai H. 2020. Asian Working Group for Sarcopenia: 2019. Journal of the American Medical Directors Association 121: 300-307.

5. Cruz-Jentoft AJ, Bahat G, Bauer J, Boirie Y, Bruyere O, Cederholm T, Cooper C, Landi F, Rolland Y, Sayer AA, Schneider SM, Sieber CC, Topinkova E, Vandewoude M, Visser M, Zamboni M. 2019. Sarcopenia: revised European consensus on definition and diagnosis. Age Ageing 48(1): 16-31. doi: 10.1093/ageing/afy169

6. Fahal IH. Uremic sarcopenia: aetiology and implications. 2014. Nephrology Dialysis Transplantation 29(9): 1655-1665, https://doi.org/10.1093/ndt/gft070

7. Fujita S, Rasmussen BB, Cadenas JG, Grady JJ, Volpi E. 2006. Effect of insulin on human skeletal muscle protein synthesis is modulated by insulin-induced changes in muscle blood flow and amino acid availability. American Journal of Physiology Endocrinology and Metabolism 291(4): E745-E754. doi: 10.1152/ajpendo.00271.2005

8. Gregg LP, Carmody T, Le D, Martins G, Trivedi M, Hedayati SS. 2020. A systematic review and meta-analysis of depression and protein-energy wasting in kidney disease. Kidney International Reports 5(3): 318-330. doi: 10.1016/j.ekir.2019.12.009

9. Gomes EP, Reboredo MM, Carvalho EV, Teixeira DR, Carvalho LF, Filho GF, de Oliveira JC, Sanders-Pinheiro H, Chebli JM, de Paula RB, Pinheiro Bdo V. 2015. Physical activity in hemodialysis patients Measured by Triaxial Accelerometer. Biomed Research International 2015:645645. doi: 10.1155/2015/645645

10. Ingsathit A, Thakkinstian A, Chaiprasert A, Sangthawan P, Gojaseni P, Kiattisunthorn K, Ongaiyooth L, Vanavanan S, Sirivongs D, Thirakhupt P, Mittal B, Singh AK. 2010. Prevalence and risk factors of chronic kidney disease in the Thai adult population: Thai SEEK study. Nephrology Dialysis Transplantation 25(5): 1567-1575. doi: $10.1093 /$ ndt/gfp669

11. Kanjanabuch T, Takkavatakarn K. 2020. Global Dialysis Perspective: Thailand. Kidney360 1 (7): 671-675. DOI: https://doi.org/10.34067/KID.0000762020

12. Kim JK, Choi SR, Choi MJ, Kim SG, Lee YK, Noh JW, Kim HJ, Song YR. 2014. Prevalence of and factors associated with sarcopenia in elderly patients with end-stage renal disease. Clinical Nutrition 33:64-68.

13. Kim TN, Park MS, Yang SJ, Yoo HJ, Kang HJ, Song W, Seo JA, Kim SG, Kim NH, Baik SH, Choi DS, Choi KM. 2010. Prevalence and determinant factors of sarcopenia in patients with type 2 diabetes: the Korean Sarcopenic obesity study (KSOS). Diabetes Care 33:1497-1499. doi: 10.2337/dc09-2310.

14. Krittayaphong R, Rangsin R, Thinkhamrop B, Hurst C, Rattanamongkolgul S, Sripaiboonkij N, Wangworatrakul W. 2017. Prevalence of chronic kidney disease 
300

301

302

303

304

305

306

307

308

309

310

311

312

313

314

315

316

317

318

319

320

321

322

323

324

325

326

327

328

329

330

331

332

333

334

335

336

337

338

339

340

341

342

343

344

345

associated with cardiac and vascular complications in hypertensive patients: A multicenter, nation-wide study in Thailand. BMC Nephrol 18(1): 115. doi: 10.1186/s12882-017-0528-3.

15. Mori K, Nishide K, Okuno S, Shoji T, Emoto M, Tsuda A, Nakatani S, Imanishi Y, Ishimura E, Yamakawa T, Shoji S, Inaba M. 2019. Impact of diabetes on sarcopenia and mortality in patients undergoing hemodialysis. BMC Nephrology 20: 105.doi: $10.1186 / \mathrm{s} 12882-019-1271-8$

16. Nishi H, Takemura K, Higashihara T, Inagi R. 2020. Uremic sarcopenia: clinical evidence and basic experimental approach. Nutrients 12(6): 1814.doi: 10.3390/nu12061814

17. Park SW, Goodpaster BH, Strotmeyer ES, de Rekeneire N, Harris TB, Schwartz AV, Tylavsky FA, Newman AB. 2006. Decreased muscle strength and quality in older adults with type 2 diabetes: the health, aging, and body composition study. Diabetes 55:18131818. doi: $10.2337 / \mathrm{db} 05-1183$.

18. Rasmussen BB, Fujita S, Wolfe RR, Mittendorfer B, Roy M, Rowe VL, Volpi E. 2006. Insulin resistance of muscle protein metabolism in aging. FASEB Journal 20(6):768-9. doi: 10.1096/fj.05-4607fje.

19. Ren H, Gong D, Jia F, Xu B, Liu Z. 2016. Sarcopenia in patients undergoing maintenance hemodialysis: incidence rate, risk factors and its effect on survival risk. Renal Failure 38(3):364-371. doi: 10.3109/0886022X.2015.1132173.

20. Sabatino A, Cuppari L, Stenvinkel P, Lindholm B, Avesani CM. 2020. Sarcopenia in chronic kidney disease: what have we learned so far? Journal of Nephrology 2. doi.org/10.1007/s40620-020-00840-y

21. Schmidt LL, Karabin BL, Malone AC. 2017. Postural orthostatic tachycardia syndrome (POTS): Assess, diagnoses, and evaluate for POTS treatment (ADEPT). Integr Med Int 4:142-153.

22. Takemura K, Nishi H, Inagi R. 2020. Mitochondrial Dysfunction in Kidney Disease and Uremic Sarcopenia. Frontiers in Physiology 11: 565023. doi: 10.3389 /fphys.2020.565023

23. Vettoretti S, Caldiroli L, Armelloni S, Ferrari C, Cesari M, Messa P. 2019. Sarcopenia is associated with malnutrition but not with systematic inflammation in older persons with advanced CKD. Nutrients 11(6):1378. doi: 10.3390/nu11061378.

24. Wang T, Feng X, Zhou J, Gong H, Xia S, Wei Q, Hu X, Tao R, Li L, Qian F, Yu L. 2016. Type 2 diabetes mellitus is associated with increased risks of sarcopenia and presarcopenia in Chinese elderly. Scientific Reports 6:38937. doi: 10.1038/srep38937.

25. Whelton, PK Carey RM, Aronow WS, CaseyJr DE, Collins KJ, Himmelfarb CD, DePalma SM, Gidding S, Jamerson KA, Jones DW, MacLaughlin EG, Muntner P, Ovbiagele B, SmithJr SC, Spencer CC, Stafford RS, Taler SJ, Thomas RJ, WilliamsSr KA, Williamson JD, WrightJr JD. 2018. 2017 ACC/AHA/AAPA/ABC/ACPM/AGS/APhA/ASH/ASPC/NMA/PCNA Guideline for the Prevention, Detection, Evaluation, and Management of High Blood Pressure in Adults: A Report of the American College of Cardiology/American Heart Association Task Force on Clinical Practice Guidelines. Hypertension 71(6): e13-e115.

26. Yuenyongchaiwat K. \& Boonsinsukh R. Sarcopenia and its relationships with depression, cognition, and physical activity in Thai community-dwelling elderly. Current Gerontology and Geriatrics Research 2020: Article ID 8041489 


\section{Figure 1}

\section{Diagnostic algorithms}

Figure 1. Diagnostic algorithms of Asian Working Group for Sarcopenia in chronic kidney disease 


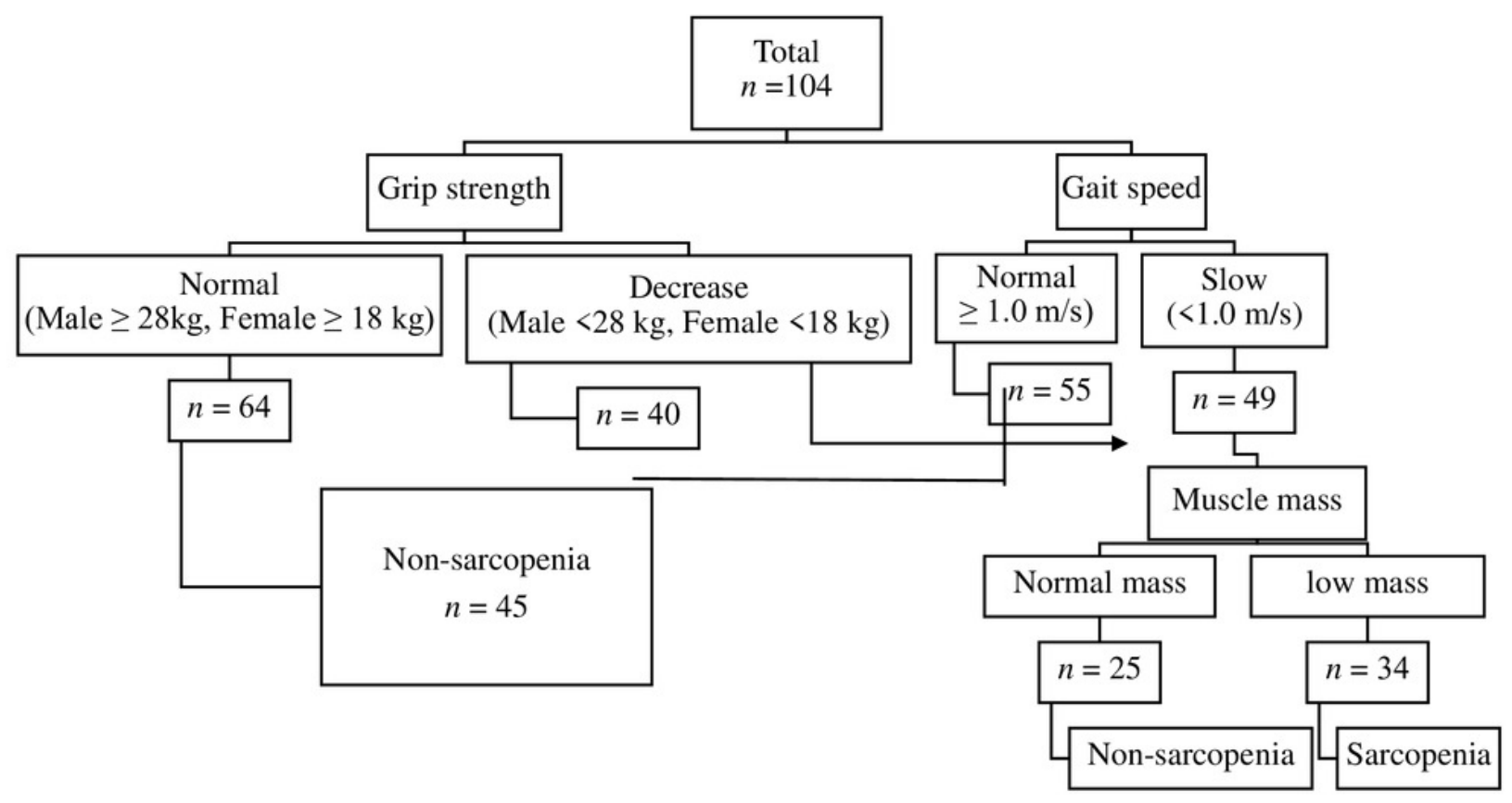




\section{Table $\mathbf{1}$ (on next page)}

Table 1 Characteristic data

Characteristic data of the participating hemodialysis patients with statistical analysis of differences between sarcopenic and non-sarcopenic patients 
1 Table 1. Characteristic data of the participating hemodialysis patients with statistical analysis of 2 differences between sarcopenic and non-sarcopenic patients

3

\begin{tabular}{|c|c|c|c|c|c|c|}
\hline & $\begin{array}{c}\text { Total } \\
(n=104)\end{array}$ & $\begin{array}{l}\text { Sarcopenia } \\
\quad(n=34)\end{array}$ & $\begin{array}{c}\text { No sarcopenia } \\
(n=70)\end{array}$ & t (102) & $P$-value & $95 \% \mathrm{CI}$ \\
\hline & Mean(SD) & Mean(SD) & Mean(SD) & & & \\
\hline Sex & & & & $0.021 *$ & 1.000 & 0.414 to 2.138 \\
\hline Male $(\%)$ & $54(51.92)$ & $18(17.31)$ & $36(34.62)$ & & & \\
\hline Female $(\%)$ & $50(48.08)$ & $16(15.38)$ & $34(32.69)$ & & & \\
\hline Age (years) & $59.74 \pm 13.62$ & $68.65 \pm 13.89$ & $55.41 \pm 11.25$ & 5.20 & $<.001$ & 8.188 to 18.277 \\
\hline \multicolumn{7}{|l|}{ Underlying diseases } \\
\hline Hypertension (\%) & $74(71.15)$ & $22(21.15)$ & $52(50.00)$ & $1.023 *$ & .359 & 0.262 to 1.537 \\
\hline Diabetes mellitus (\%) & $38(37.54)$ & $18(17.31)$ & $20(19.23)$ & $5.861 *$ & .018 & 1.202 to 6.580 \\
\hline Dyslipidemia (\%) & $24(23.08)$ & $7(6.73)$ & $17(16.35)$ & $0.176^{*}$ & .806 & 0.299 to 2.186 \\
\hline Gout (\%) & $11(10.58)$ & $4(3.85)$ & $7(6.73)$ & $0.075^{*}$ & .747 & 0.326 to 4.417 \\
\hline Heart disease $(\%)$ & $23(22.12)$ & $9(8.65)$ & $14(13.46)$ & $0.556^{*}$ & .461 & 0.551 to 3.765 \\
\hline BMI $\left(\mathrm{kg} / \mathrm{m}^{2}\right)$ & $23.48(4.11)$ & $21.11(2.47)$ & $24.64(4.26)$ & -4.471 & $<.001$ & -5.094 to -1.963 \\
\hline Duration of hemodialysis (yrs.) & $5.86(4.95)$ & $7.41(5.70)$ & $5.10(4.39)$ & 2.280 & .025 & 0.301 to 4.325 \\
\hline Gait speed $(\mathrm{m} / \mathrm{s})$ & $0.96(0.35)$ & $0.73(0.35$ & $1.07(0.29)$ & -5.238 & $<.001$ & -0.468 to -0.211 \\
\hline Grip strength $(\mathrm{kg})$ & $23.30(8.41)$ & $17.26(5.63)$ & $26.23(7.98)$ & -5.878 & $<.001$ & -11.997 to -5.943 \\
\hline Skeletal mass index $\left(\mathrm{kg} / \mathrm{m}^{2}\right)$ & $6.21(1.29)$ & $5.18(0.95)$ & $6.71(1.12)$ & -6.846 & $<.001$ & -1.975 to -1.088 \\
\hline Depression scores & $7.43(6.35)$ & $10.29(7.19)$ & $6.04(5.43)$ & 3.359 & .001 & 1.741 to 6.761 \\
\hline Physical activity (MET minutes per week) & $1882.31(2946.57)$ & $592.06(662.66)$ & $2535.73(3383.55)$ & -3.408 & .001 & -3161.93 to -835.406 \\
\hline
\end{tabular}

5 BMI; body mass index 
Table 2 (on next page)

Table 2 Correlation of risk factors with sarcopenia

Correlation of risk factors with sarcopenia 
1 Table 2. Correlation of risk factors with sarcopenia

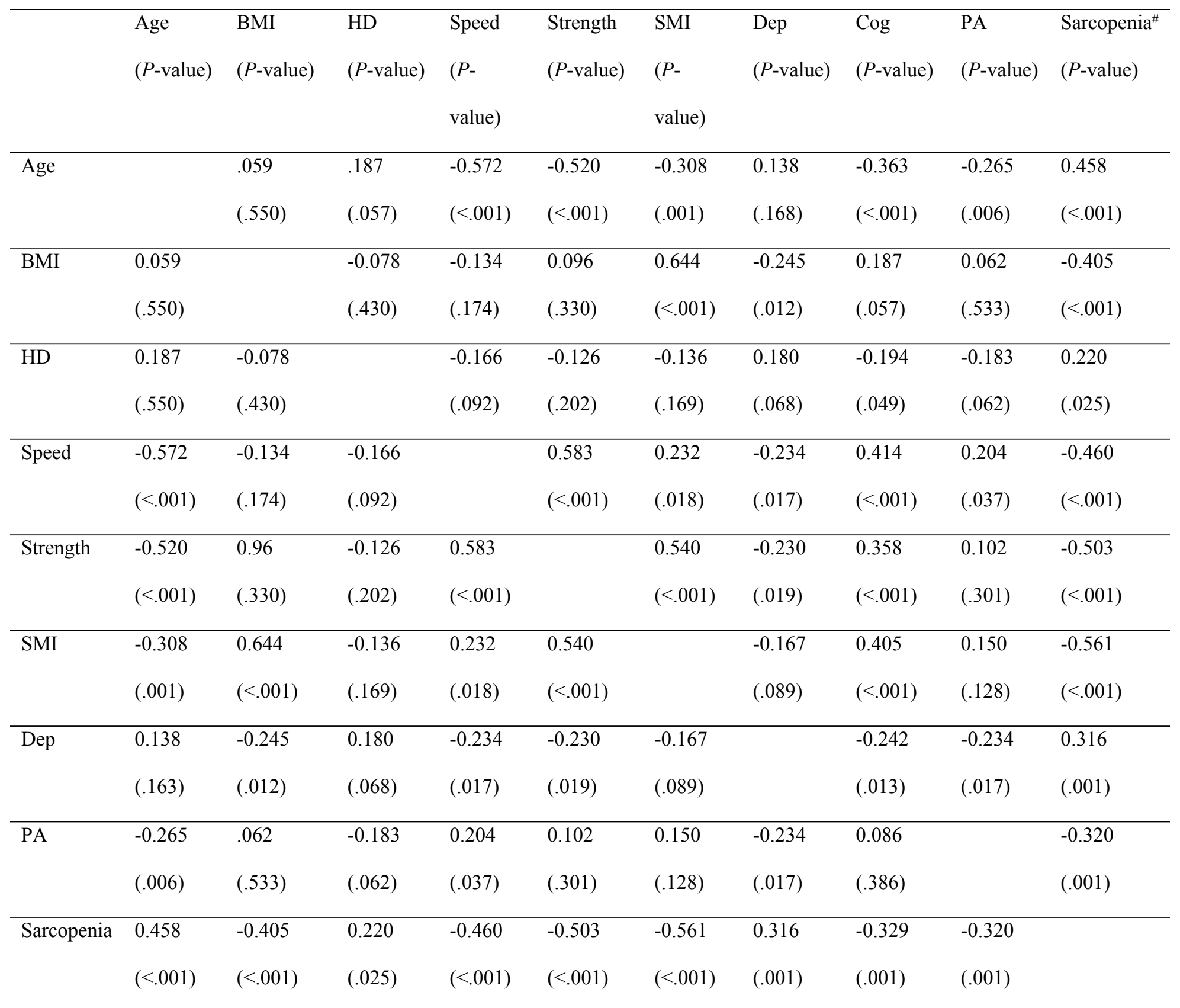

2

3 \# Sarcopenia: 0 defined as no sarcopenia, 1 defined as sarcopenia, BMI: body mass index, SMI:

4 skeletal muscle index, HD: duration of hemodialysis, Dep: depression, Cog: cognitive

5 performance, PA: physical activity 


\section{Table 3 (on next page)}

Table 3 Results of logistic regression analysis of risk factors for sarcopenia

Results of logistic regression analysis of risk factors for sarcopenia 
3 Table 3. Results of logistic regression analysis of risk factors for sarcopenia

\begin{tabular}{|c|c|c|c|c|}
\hline Risk factors & OR (95\% CI) & $P$-value & OR $(95 \%$ CI) & $P$-value \\
\hline Age & \multicolumn{4}{|l|}{ Reference $<60$ years } \\
\hline Age $\geq 60$ years & $0.193(0.076-0.488)$ & \multicolumn{3}{|l|}{.001} \\
\hline Sex & \multicolumn{4}{|l|}{ Reference female } \\
\hline Male & $0.941(0.414-2.138)$ & \multicolumn{3}{|l|}{.885} \\
\hline Comorbidities & \multicolumn{4}{|c|}{ Reference no history of diabetes mellitus } \\
\hline Diabetes mellitus & $2.812(1.202-6.580)$ & .017 & $0.483(0.195-1.198)$ & .116 \\
\hline Duration of HD & \multicolumn{4}{|c|}{ Reference duration $<5$ years } \\
\hline Duration $5-10$ & $1.614(0.553-4.713)$ & .381 & $1.613(0.522-4.981)$ & .406 \\
\hline Duration $\geq 10$ years & $0.316(0.111-0.896)$ & .030 & $0.366(0.120-1.113)$ & .077 \\
\hline Depression & \multicolumn{4}{|c|}{ Reference normal range ( $0-13$ scores) } \\
\hline Depression $>14$ scores & $3.229(1.139-9.157)$ & .028 & $0.281(0.090-0.880)$ & .029 \\
\hline Physical activity & \multicolumn{4}{|c|}{ Reference moderate to high physical activity } \\
\hline Low physical activity & $4.921(2.044-11.850)$ & $<.001$ & $0.277(0.110-0.699)$ & .007 \\
\hline
\end{tabular}

\# adjusted by age

5 HD; hemodialysis 